\title{
COMPARATIVE HISTOCHEMICAL EVALUATION OF MUCINS EXPRESSION IN FETAL ESOPHAGUS AND ADENOCARCINOMAS OF THE GASTROESOPHAGEAL JUNCTION
}

\author{
S. Vernygorodskyi*, T. Rekun, P. Zhuchenko \\ Department of Pathological Anatomy, Forensic Medicine and Law, National Pirogov Memorial Medical \\ University, Vinnytsia, Ukraine
}

\begin{abstract}
Aim: To study and compare the expression patterns of mucins in the fetal gastroesophageal junction (GEJ) region and adenocarcinomas of the GEJ in adults using histochemical method. Material and Methods: To reveal the expression of different mucins, tissue sections from formalin-fixed paraffin-embedded tissue blocks of fetal GEJ and carcinomas of GEJ were stained by the following histological stains: hematoxylin and eosin, periodic acid-Schiff (PAS), alcian blue (AB), pH 2.5, combined AB-PAS, and combined AB-Aldehyde Fuchsin (AB/AF). Results: Expression of mucins within the developing fetal GEJ has been demonstrated (gestational age 5-38 weeks). We studied histochemical features of neoplastic cells in carcinomas of GEJ from 90 adult patients. The results showed that in gastric type expression of neutral mucins dominated especially in well differentiated (G1) adenocarcinomas and corresponded to the last three months of GEJ development, while in the intestinal and mixed type significant acid mucins expression was detected in moderate (G2) and poorly (G3) differentiated adenocarcinomas and corresponded to 17-25 weeks of GA. No significant coincidence in mucins expression was observed in signet ring cell carcinomas in relation to GA. Conclusion: Our data suggest that decrease of neutral mucins level can be considered reliable phenotypic marker of poor prognosis of GEJ adenocarcinomas. Study of the developmental expression of mucin genes may improve understanding of the malignant transformation of esophageal tissue. Key Words: mucin expression, gastroesophageal junction, adenocarcinoma.
\end{abstract}

The incidence of adenocarcinoma of the stomach distal to the gastric cardia is progressively declining in developed countries [1]. In contrast to gastric cancer, esophageal adenocarcinoma has recently displayed a striking increase in incidence, as reported in several countries, including the USA and Ukraine [2-4].

Recent developments in mucin histochemistry and immunohistochemistry have made reliable determination of the gastric and intestinal phenotypes of gastric carcinoma cells possible. Phenotypic expression changes from gastric epithelial cell type to intestinal epithelial cell type with the growth of gastric tumors in $\mathrm{C} 3 \mathrm{H} / \mathrm{HeN}<-->\mathrm{BALB} / \mathrm{c}$ chimeric mice treated with $\mathrm{N}$-methylN-nitrosourea [5].

The risk of adenocarcinoma developing of the esophagus is associated with glandular metaplasia of multilayer epithelium, the definition of such a morphological status is defined by the concept of Barrett's esophagus (BE). A huge number of works devoted to the BE [6-8] has not yet solved the problem of its diagnosis and prognosis. So, differential diagnosis of $\mathrm{BE}$ and monitoring of these patients remain an urgent problem. One of the solutions to this problem can be the detection of mucins expressed by neoplastic cells in adenocarcinomas.

The mucus barrier is an important factor in the protection of the esophagus from damage during deglutition and episodes of gastroesophageal reflux $[9,10]$.

Submitted: January 31, 2018.

${ }^{\star}$ Correspondence: E-mail: medservervit@gmail.com Abbreviations used: BE - Barrett's esophagus; CCLLE - columnar cell lined lower esophagus; $C R L$ - crown-rump length; GA - gestational age; GEJ - gastroesophageal junction; SFE superficial-foveolar epithelium.
It is now well known that tumors share several morphological and ultra-structural features with embryonic cells [11]. The oncogerminative theory of tumorogenesis considers tumor formation as a dynamic self-organizing process that mimics a self-organizing process of early embryo development [12].

That's why our purpose was to estimate and compare mucins secretion in the epitheliocytes of the fetal gastroesophageal junction (GEJ) and carcinoma cells so as to understand better a behavior of GEJ tumors depending on their differentiation.

\section{MATERIALS AND METHODS}

Patients. The current study was carried out on 169 autopsied formalin-fixed human embryos and fetuses of gestational age (GA) 6-38 weeks of Caucasian ethnic origin. All autopsies were performed between 4 and $24 \mathrm{~h}$ after death. The presence of congenital malformations involving the gastrointestinal tract was considered as an exclusion criterion. Crown-rump length ( $C R L$ ) was the main criterion for estimating fetal age. The fetal ages are indicated in Table 1.

\begin{tabular}{|c|c|c|c|}
\hline № & Age, weeks & Number & $\mathrm{CRL}, \mathrm{mm}$ \\
\hline 1 & $4-5$ & 6 & $6.10 \pm 0.30$ \\
\hline 2 & $6-7$ & 6 & $15.10 \pm 0.60$ \\
\hline 3 & $8-9$ & 7 & $19.40 \pm 0.50$ \\
\hline 4 & $10-11$ & 10 & $39.02 \pm 0.50$ \\
\hline 5 & $12-13$ & 11 & $58.72 \pm 2.27$ \\
\hline 6 & $14-15$ & 12 & $93.11 \pm 5.10$ \\
\hline 7 & $16-17$ & 15 & $122.17 \pm 2.70$ \\
\hline 8 & $18-20$ & 19 & $152.90 \pm 3.50$ \\
\hline 9 & $21-24$ & 20 & $192.14 \pm 1.80$ \\
\hline 10 & $25-28$ & 13 & $230.96 \pm 3.10$ \\
\hline 11 & $29-32$ & 14 & $264.83 \pm 1.70$ \\
\hline 12 & $33-36$ & 20 & $302.92 \pm 1.40$ \\
\hline 13 & $37-38$ & 16 & $341.50 \pm 5.70$ \\
\hline Total & & 169 & - \\
\hline
\end{tabular}


A total of 90 adult patients attending gastroenterology services at Vinnytsia Regional Oncology Clinical Dispensary from 2015 to 2017 were enrolled in this comparative study. All patients were thoroughly informed about the study that was approved by the local ethics committee.

There were 55 male $(61 \%)$ and 35 female patients (39\%). The mean age was $58.50 \pm 1.18$ years (range from 36 to 90 ). The surgical specimens of GEJ adenocarcinomas as well as adjacent mucosa were investigated: 78 adenocarcinomas and 12 signet-ring cell carcinomas, a distribution of patients according to TNM staging and degree of differentiation is given in Tables 2 and 3 . There were 78 resections of the esophagus for adenocarcinoma: 27 cardiac adenocarcinomas ( 16 men and 11 woman), 51 adenocarcinomas arising in columnar cell lined lower esophagus (CCLLE), 39 men and 12 woman. The difference between cardiac and CCLLEadenocarcinoma was determined by the presence of columnar epithelium between the carcinoma and either the squamous epithelium or the cardiac line. Dysplasia was estimated by using the Vienna classification of gastrointestinal epithelial neoplasia [13]. All sections were examined by three independent investigators.

Table 2. Distribution of patients with cancer of the GEJ according to degree of differentiation

\begin{tabular}{lc}
\hline \multicolumn{1}{c}{ Degree of differentiation } & Number of cases, \% \\
\hline Poorly differentiated adenocarcinoma & 29 \\
Moderately differentiated adenocarcinoma & 36 \\
Well differentiated adenocarcinoma & 22 \\
Signet-ring cell carcinoma & 13 \\
\hline
\end{tabular}

Table 3. Distribution of patients with cancer of the GEJ according to TNM staging

\begin{tabular}{|c|c|}
\hline TNM staging & Number of cases, $\%$ \\
\hline$\overline{\text { IA (T1N0M0) }}$ & 10 \\
\hline IB (T2NOMO) & 34 \\
\hline II (T3NOMO) & 15 \\
\hline IIIA (T3N1M0) & 9 \\
\hline IIIB (T3N2M0) & 9 \\
\hline IV (T4N1M0) & 5 \\
\hline IV (T4N2M0) & 4 \\
\hline IV (T3N2M1) & 3 \\
\hline IV (T4N2M1) & 4 \\
\hline
\end{tabular}

Histochemistry. The material consisted of biopsy and surgical samples. All the biopsy samples and surgical specimens were fixed in $10 \%$ neutral buffered formalin for $48 \mathrm{~h}$ and embedded in paraffin. Serial sections, $4 \mu \mathrm{m}$ thick, were cut from each sample, and stained by the following stains: haematoxylin and eosin, $A B \mathrm{pH}$ 2.5-PAS to differentiate acid from neutral mucins, Gomory aldehyde fuchsin/AB pH 2.5 to differentiate sulphomucins from sialomucins (Table 4).

Table 4. Histochemical techniques used to characterize mucins

\begin{tabular}{lll}
\multicolumn{1}{c}{ Mucin type } & \multicolumn{1}{c}{ AB-PAS } & AF-AB \\
\hline Neutral mucins & Magenta & 0 \\
Sialomucins & Blue & Blue \\
Sulphomucins & Blue & Purple \\
\hline
\end{tabular}

Note: 0 - negative staining; PAS - periodic acid Schiff; AB - alcian blue; $\mathrm{AF}$ - aldehyde fuchsin.

The results were expressed semiquantitatively for each histological group as the number of sections positively stained, the predominant cell type labeled, and the average score of the positively labeled cells. The extent of staining for each mucins, was scored according to the number of cytoplasmic and luminal stained carcinoma cells in 100 tumor cells. Less than $5 \%$ positive cells was accepted as negative, while $\geqslant 5 \%$ positive cells was accepted as positive [14]. The microscopy and photographing of histological specimens were performed by using an optical microscope OLIMPUS BX 41 with magnifications of 40, 100, 200 and 400 . The images were processed with standard phase analysis; morphometry and statistical processing were performed using the Quick PHOTO MICRO 2.3 program. The content of cellular elements was determined on the basis of a unit of conventional area $\left(0.01 \mathrm{~mm}^{2}\right)$.

Statistical analysis. Results of histochemical alterations were compared to the clinicopathologic features using chi-square test with two tailed $p$ value, $p<0.05$ was considered as significant.

\section{RESULTS AND DISCUSSION}

In embryos with CRL $6.1 \pm 0.3 \mathrm{~mm}$ (4-5 gestation weeks), the epithelium of GEJ becomes three-layered. Its deepest layer is formed by columnar cells with basophilic cytoplasm and scant cytoplasm that contains predominantly neutral mucins $(4.85 \pm 0.31 \%)$ without sulfomucins and small amount of acid mucins (Fig. 1, 2).

The results of our research differ from those of other authors whose data testify to the fact that cytochemical changes are not typical of the given period of observation [14]. In our research, we noted the appearance of bright pink and crimson granules in subnuclear parts of the cytoplasm of the three-layered columnar epithelium, which was the evidence of the presence of neutral mucins and glycogen already in this period. The surrounding submucosa displayed significant alcianophylia, which was well noticeable while using the combined $\mathrm{PAS}$ reaction with $\mathrm{AB}$.

In embryos with CRL $15.1 \pm 0.6 \mathrm{~mm}$ (6-7 gestation weeks), the epithelium remains three-layered with the presence of up to $5.18 \pm 0.3 \%$ of neutral mucins in the cellular cytoplasm of the mucous membrane of GEJ and small amount of sulphated mucins in the gastric part of GEJ (Fig. 1). Already in this period, we observed the appearance of single vacuoles in the cells cytoplasm which had not been registered by other authors [15]. By the eighth week (CRL 19.4 $\pm 0.5 \mathrm{~mm}$ ), the epithelium remains pseudostratified. However, already in the ninth week, the vacuoles reach their largest volumes.

After the tenth week (CRL 37-38 mm), a pseudostratified epithelium starts to get considerably thinner, and starting with CRL $40 \mathrm{~mm}$ becomes ciliated multilayered columnar. We observed decrease of acid mucins in the esophageal epithelium and increase their level in gastric epithelium. Starting with the twelfth week, superficial-foveolar epithelium (SFE) of the stomach cardiac region contains both neutral and acid mucins with the prevailing of the latter. The verification of mucins found out the heterogeneity of the mucous secrete in the apical part of SFE. That is, the internal part of the secrete got rose-pink color, while the external one - blue (this part contained 


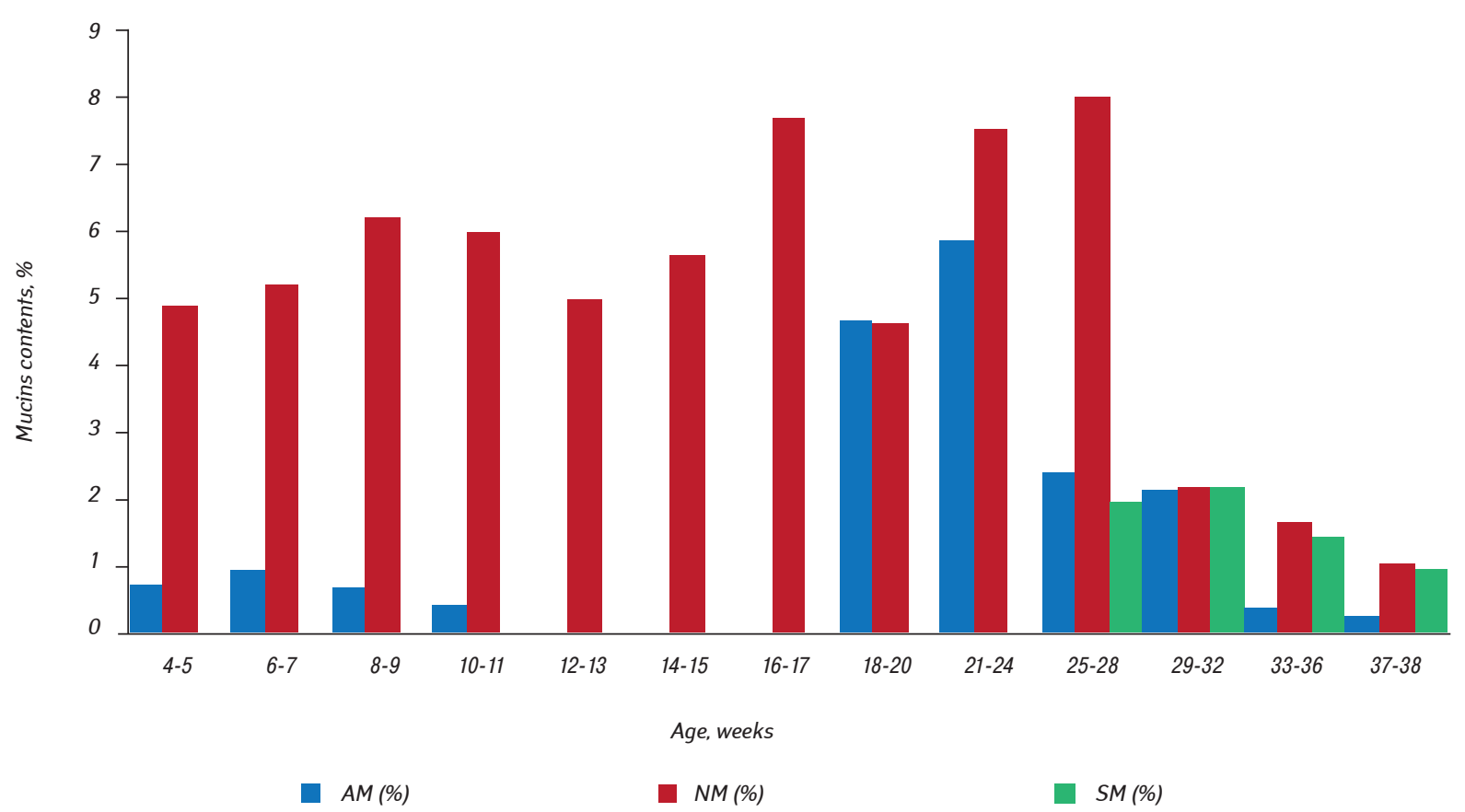

Fig. 1. Content of mucins in esophageal epitheliocytes according to the phase image analysis data based on $0.01 \mathrm{~mm}^{2}$ Note: AM - acid mucins; NM - neutral mucins; SM - sulfomucins.

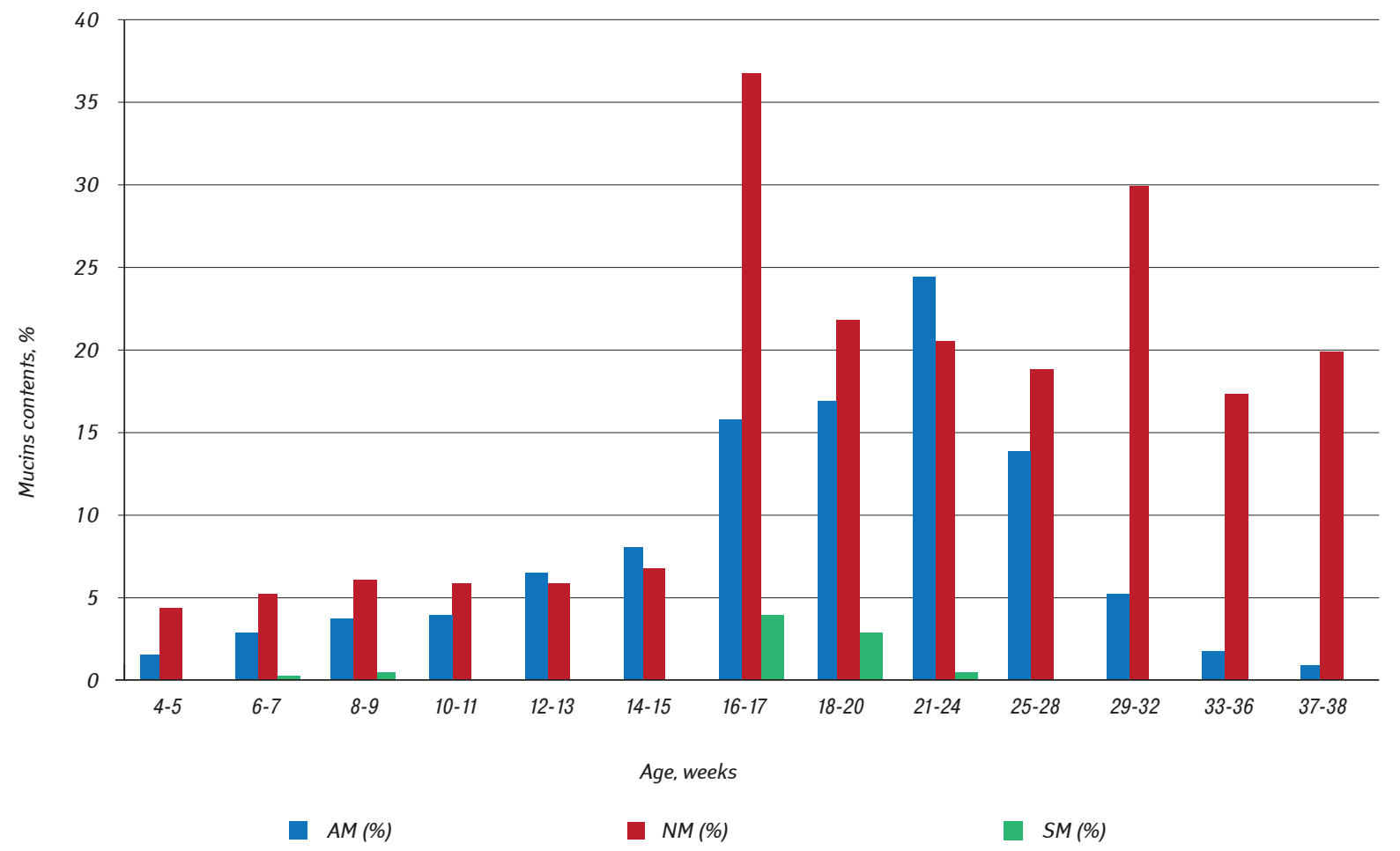

Fig. 2. Content of mucins in cardiac epitheliocytes according to the phase image analysis data based on $0.01 \mathrm{~mm}^{2}$ Note: AM - acid mucins; NM - neutral mucins; SM - sulfomucins.

acid mucins). As it can be seen from Fig. 1, in this period the number of neutral mucins remains on the same level up to sixteenth week. However, starting with the sixteenth week, when squamous epithelium arises in the lower part of the esophagus and columnar epithelium of the stomach cardia part becomes more differentiated, their number veritably increases up to $15.9 \pm 0.1$ and $37.0 \pm 0.4 \%$, respectively (Fig. 1, 2) and also sulfomucins appear in gastric epithelium again. From 25 to 38 gestation weeks, the progressing decrease of acidic and sulphated mucins is observed, while the number of neutral ones increases.

During the last three months of the gestational period, the content of acid mucins substantially decreases but sulphated mucins do not technically occur in gastric epitheliocytes, SFE and the glandular epithelium containing mainly neutral mucins. The esophageal epithelium during these last three months is charac- 
terized by reduction of acid mucins with small amount of sulfomucins and predominance of neutral mucins.

In the presence of adenocarcinomas, the intensity of the PAS reaction decreases from marked to weak. In this the distribution of secrete is predominantly granular. Poorly differentiated adenocarcinoma (G3) showed mainly the intestinal and mixed phenotype, while 55\% of the well differentiated adenocarcinomas (G1) had a gastric phenotype. Three of G3 adenocarcinomas were negative for all types of mucins and we applied them to $\mathrm{N}$-phenotype. Table 5 shows the distribution of histological diagnosis by secretory status.

The proportion of gastric phenotype of GEJ adenocarcinomas is dominated by neutral mucins in G1 adenocarcinomas and according to the qualitative properties corresponds to last three gestation weeks of GEJ development, while the intestinal type includes mainly acid mucins expression by neoplastic cells of moderately differentiated (G2) and G3 adenocarcinomas. Mixed phenotype tumors present intermediate patterns. The sulfomicins expression was revealed predominantly in G2 or G3 adenocarcinomas and signet ring carcinomas. Intestinal type of adenocarcinomas was characterized by a mucin pattern similar to that which we have observed in the intestinal metaplasia of the stomach [16]. Ten of these studies included the co-expression of the acid mucins and sulfomucins.

We have to note, however, that in the presence of the secretion of mucins, the production of acid mucins was revealed more often as compared to sulfomucins, which is more characteristic for the intestinal type of cancer. These results differ from those of some researchers [17, 18], who mainly identified sulfomucins in the mentioned parts of the gastric mucosa. As opposed to this, all three types of mucins, acid, neutral and sulfomucins were found out in signet ring cell carcinomas.

Epithelial dysplasia was observed in 13 of 51 patients (25\%) with CCLLE: low-grade dysplasia was found in 9 patients ( 6 men and 3 women) and highgrade dysplasia in 4 ( 4 men and 3 women). All these cases were found in intestinal-type epithelium, and the association was statistically significant $(p<0.05)$. In all nine cases with low-grade epithelial dysplasia, a loss of mucin in the cytoplasm of the goblet cells was observed, with a prevalence of sialomucins over sulphomucins. Neutral mucins were always absent. In the adjacent squamous epithelium without metaplastic and dysplastic changes the sulphomucins secretion was poor but neutral mucins were most common compared with acid ones.

The data obtained evidence that morphological heterogeneity is typical for both GEJ adenocarcinomas and gastric ones [19]. It is necessary to note that in dif- ferent patients various combinations of these types are observed. These data coincide with the research of Yamamoto et al. (2005), and our previous investigation $[16,20]$ demonstrating the presence of neutral gastric mucins in the mucous area preserved around G1 adenocarcinomas, and we suggest that the majority of GEJ carcinomas retain gastric differentiation, particularly in $\mathrm{G} 1$ adenocarcinomas.

Based on the results of our studies, as well as data obtained by other authors [21], we can suggest a significant clarification to the existing histological classification of the adenocarcinomas of the GEJ:

- a gastric (G) epithelial cell type (cells secretions of which contain mainly neutral mucins and revealed by PAS-reaction);

- an intestinal (I) epithelial cell type (cells secretions of which contain mainly acid mucins, revealed by $A B$ );

- a mixed (Gl) type (cells secretions of which contain both neutral and sialomucins which are distinctly revealed in the combined stain $\mathrm{AB}$ pH 2.5 - PASreaction);

- a null phenotype ( $\mathrm{N}$-phenotype), neoplastic cells with negative reaction to any of the stains (negative in the combined stain $\mathrm{AB} \mathrm{pH} 2.5$ - PAS-reaction). The data of the histochemical research enabled to state that $\mathrm{G} 1$ adenocarcinomas of the gastric type correspond to the last three months of GEJ development, while G2 and G3 adenocarcinomas to 17-25 weeks, when the transdifferentiation of the columnar epithelium into stratified ones takes place.

The mixed type was mainly observed in 18-week fetuses, which corresponds to the appearance of the alcianophylic gastric epithelium in the GEJ. However, in the course of time, alcianophylia is lost and neutral mucins begin to prevail in both the superficial and glandular epithelium. Our data suggest that embryogenesis and cancer share several common critical pathways.

Thus, the results of our research showed the decrease of the part of neutral mucins in the gastric type of G1 and G2 adenocarcinomas, and their almost complete disappearance in the case of G3 adenocarcinoma development. Analogous changes were observed also in the mucosa of the GEJ surrounding adenocarcinomas.

In contrary to adenocarcinomas, in signet ring cell carcinomas no correspondence between the mucosal qualitative changes and the gestational period was revealed. In most cases, the mixed type of mucosa secretions dominated, which proves the high heterogeneity of the given cancer form and enables to recommend a wider use of the combined PAS reaction with $A B$ in clinical practice.

Table 5. Content of mucins in neoplastic cells according to the phase image analysis data based on $0.01 \mathrm{~mm}^{2}$

\begin{tabular}{|c|c|c|c|c|c|}
\hline $\begin{array}{c}\text { Tumor, histological } \\
\text { type, }(n)\end{array}$ & $\mathrm{AM}, \%(\mathrm{n})$ & $\mathrm{NM}, \%(\mathrm{n})$ & MM, \% (n) & $\mathrm{SM}, \%(\mathrm{n})$ & $\begin{array}{l}\text { Negative for all } \\
\text { mucins, } n\end{array}$ \\
\hline $\mathrm{G} 1(20)$ & $6.71 \pm 0.23(7)$ & $11.25 \pm 0.56(11)$ & $4.45 \pm 0.19(2)$ & - & - \\
\hline G2 (32) & $3.04 \pm 0.31^{*}(10)$ & $6.65 \pm 0.36^{*}(15)$ & $3.3 \pm 0.18^{\star}(5)$ & $3.5 \pm 0.3(2)$ & - \\
\hline G3 $(26)$ & $1.56 \pm 0.29^{*}(9)$ & $0.58 \pm 0.08 *(5)$ & $2.06 \pm 0.26^{\star}(8)$ & $1.49 \pm 0.21(4)$ & 3 \\
\hline Signet-ring carcinoma (12) & $34.75 \pm 0.25^{*}(2)$ & $17.5 \pm 0.5^{\star}(2)$ & $17.06 \pm 0.45^{\star}(4)$ & $6.08 \pm 0.31(4)$ & - \\
\hline
\end{tabular}

Note: G1 - well differentiated adenocarcinomas; G2 - moderately differentiated adenocarcinomas; G3 - poorly differentiated adenocarcinomas; AM - acid mucins; NM - neutral mucins; MM - mixed mucins (acid+neutral); SM - sulfomucins; - absence of mucins; * $p<0.05$ compared to G1; $\mathrm{n}$ - number of cases. 


\section{REFERENCES}

1. El-Serag HB, Mason AC, Petersen N, et al. Epidemiological differences between adenocarcinoma of the oesophagus and adenocarcinoma of the gastric cardia in the USA. Gut 2002; 50: 368-72.

2. Newnham A, Quinn MJ, Babb P, et al. Trends in the subsite and morphology of oesophageal and gastric cancer in England and Wales 1971-1998. Aliment Pharmacol Ther 2003; 17: 665-76.

3. Blanca Piazuelo M, Haque S, Delgado A, et al. Phenotypic differences between esophageal and gastric intestinal metaplasia. Modern Pathol 2004; 17: 62-74.

4. Fedorenko ZP, Michailovich YYo, Goulak LO, et al. Cancer in Ukraine, 2015-2016. Ukrainian Cancer Registry Statistics. Bull Nat Cancer Registry of Ukraine (Engl) 2017; 18: C15.

5. Tatematsu M, Tsukamoto T, Inada K. Stem cells and gastric cancer: role of gastric and intestinal mixed intestinal metaplasia. Cancer Sci 2003; 94: 135-41.

6. Macke RA, Nason KS, Mukaisho K, et al. Barrett's esophagus and animal models. Ann N Y Acad Sci 2011; 1232: $392-400$.

7. Kosoff RE, Gardiner KL, Merlo LM, et al. Development and characterization of an organotypic model of Barrett's esophagus. J Cell Physiol 2012; 227: 2654-9.

8. Shilpa J, Dhingra S. Pathology of esophageal cancer and Barrett's esophagus. Ann Cardiothorac Surg 2017; 6: $99-109$.

9. Corfield AP, Carroll D, Myerscough N, et al. Mucins in the gastrointestinal tract in health and disease. Front Biosci 2001; 6: 1321-57.

10. Geboes K. Barrett's esophagus: the metaplasiadysplasia-carcinoma sequence: morphological aspects. Acta Gastroenterol Belg 2000; 63: 13-7.

11. Bizzarri M, Cucina A, Biava PM, et al. Embryonic morphogenetic field induces phenotypic reversion in cancer cells. Review Article. Curr Pharm Biotechnol 2011; 12: $243-53$
12. Vinnitsky V. The development of a malignant tumor is due to a desperate asexual self-cloning process in which cancer stem cells develop the ability to mimic the genetic program of germline cells. Intrinsically Disord Proteins 2014; 2: e29997.

13. Schlemper $R$, Riddell $R$, Kato $Y$, et al. The Vienna classification of gastrointestinal epithelial neoplasia. Gut 2000; 47: 251-5.

14. İlhan Ö, Han Ü, Önal B, et al. Prognostic significance of MUC1, MUC2 and MUC5AC expressions in gastric carcinoma. Turk J Gastroenterol 2010; 21: 345-52.

15. Hashimoto K, Tamura K, Otani H, Tanaka O. Histocytochemical and immunohistochemical studies related to the role of glycogen in human developing digestive organs. Anatom Embryol 1995; 192: 497-505.

16. Vernygorodskyi S. Immunohistochemical evaluation of mucin expression in precancerous tissue of stomach. Exp Oncol 2013; 35: 114-7.

17. Wu JF. The relationship between carcinoembryonic antigen (CEA) expression and histogenesis of gastric cancer (application of immunohistochemical and mucin histochemical techniques). Zhonghua Zhong Liu Za Zhi 1992; 14: 13-6.

18. Warson C, Van de Bovenkamp, Korteland-Van Male A, et $\boldsymbol{a l}$. Barretts esophagus is characterized by expression of gastric-type mucins (MUC 5 AC, MUC 6) and TFF peptides (TFF 1 and TFF 2), but the risk of carcinoma development may be indicated by the intestinal -type mucin MUC 2. Hum Pathol 2002; 33: 660-8.

19. Vasilenko IV, Gulkov UK, Zaporozchenco NV. Histological heterogeneity gastric carcinoma. Ukr Morphol Almanac 2011; 9: 26-31 (in Russian).

20. Yamamoto S, Kijima H, Hara T, et al. Mucin expression and proliferating cell index of esophageal Barretts adenocarcinoma. Jnt J Mol Med 2005; 16: 375-80.

21. Gwang Ha Kim, Geun Am Song, Do Youn Park, et al. Clinicopathologic significance of gastric and intestinal phenotypic marker expression in gastric carcinomas. Korean J Int Med 2005; 20: 191-7. 